\title{
Oscillation May Play a Role in Time Domain Central Auditory Processing
}

\author{
Alexander V. Galazyuk and Albert S. Feng \\ Department of Molecular and Integrative Physiology, and the Beckman Institute, University of Illinois, \\ Urbana, Illinois 61801
}

To study how sound intensity altered the temporal response pattern of a unit, we recorded from 92 single neurons in the inferior colliculus (IC) of the little brown bat and investigated their firing patterns in response to brief tone pulses $(2 \mathrm{msec}$ duration) at the characteristic frequency of the unit over a wide dynamic range (10-90 dB sound pressure level). We found two unusual response characteristics at high sound levels in approximately one-third of the IC neurons investigated. For 16 IC neurons (17\%), an increase in sound level not only elicited a shorter response latency and an increase in spike count but also transformed the firing pattern of the unit from phasic to periodic; this pattern was more pronounced at higher sound levels. The firing periodicity was unit specific, ranging from 1.3 to $6.7 \mathrm{msec}$. Twenty-seven IC neurons (29\%) exhibited a longer response latency at higher sound levels compared with lower sound levels [i.e., paradoxical latency shift (PLS)]. The majority

In echolocating bats, many neurons in the auditory midbrain and forebrain are tuned to time delays between a pair of sound pulses of unequal amplitudes, approximating the sonar signal and returning echo of a bat (Feng et al., 1978; O'Neill and Suga, 1979; Sullivan, 1982a,b; Mittmann and Wenstrup, 1995; O’Neill, 1995). Although the characteristics of the delay-tuned response have been described in detail, its underlying mechanism is unclear. Sullivan (1982a,b) proposed two models to explain delay-tuned responses of cortical neurons in the little brown bat after having observed an unusual response property in these neurons [i.e., paradoxical latency shift (PLS), which is characterized by longer response latencies for intense sounds than for weaker sounds]. He showed that PLS is central to creating temporal selectivity between a loud sonar signal and a weaker echo. In particular, the delay in the response of a unit to the loud sonar signal allows it to align temporally with the response of the unit to the weak echo, and this coincidence produces a much stronger response than the individual responses alone. In the Delay Line model, weak sound is assumed to project, via a short-latency pathway, to the cortex, whereas intense sound projects to the cortex via a long-latency pathway. These two separate pathways then converge on a corti-

\footnotetext{
Received Dec. 4, 2000; revised Mar. 9, 2001; accepted Mar. 13, 2001.

This study was supported by Grant RO1-DC00663 from the National Institute on Deafness and Other Communication Disorders of the National Institutes of Health. We thank Daniel Llano and two anonymous reviewers for their comments on earlier versions of this manuscript and thank many colleagues at the Beckman Institute for helpful discussion of the data described in this paper.

Correspondence should be addressed to Alexander V. Galazyuk, University of Illinois, Beckman Institute, 405 North Mathews Avenue, Urbana, IL 61801. E-mail: galazyuk@uiuc.edu.

Copyright (c) 2001 Society for Neuroscience $\quad 0270-6474 / 01 / 210001-05 \$ 15.00 / 0$
}

of this population showed a one or more quantum increase in latency when sound level was elevated. The quantum shift was also unit specific, ranging from 1.2 to $8.2 \mathrm{msec}$. We further investigated the firing patterns of 14 IC neurons showing PLS before, during, and after iontophoretic application of bicuculline. For 12 of these neurons, drug application abolished the PLS and transformed the firing patterns of the unit at high sound levels from phasic into sustained periodic discharges. Our results suggest that neural oscillation in combination with ordinary inhibition may be responsible for the creation of PLSs shown previously to be important for temporal information processing.

Key words: paradoxical latency shift; inferior colliculus; bat; echolocation; delay-tuned response; hearing; temporal processing

cal neuron to produce the delay-tuned response (Sullivan, 1982b, his Fig. 10). In the Timed Inhibitory model, it is assumed that an intense sound activates an inhibitory synapse; therefore, the response of the unit that results from the inhibitory rebound is delayed in time (Sullivan, 1982b, his Fig. 11). In contrast, weak sounds produce weak or no inhibition, and therefore the response occurs with no imposed inhibitory delay. Although these hypotheses are attractive, neither has been validated empirically.

Recent evidence shows that PLS is also a response characteristic of central neurons in the auditory system of mammals that do not echolocate (for review, see Klug et al., 2000) and of amphibians (D. Llano, A. V. Galazyuk, and A. S. Feng, unpublished observation). As such, PLS may be a common response feature in the vertebrate. For nonecholocating animals, however, these neurons are unlikely to be involved in echolocation; rather, they may play a role in specific temporal processing that has yet to be determined.

This paper describes physiological findings in the inferior col-

This article is published in The Journal of Neuroscience, Rapid Communications Section, which publishes brief, peerreviewed papers online, not in print. Rapid Communications are posted online approximately one month earlier than they would appear if printed. They are listed in the Table of Contents of the next open issue of JNeurosci. Cite this article as: JNeurosci, 2001, 21:RC147 (1-5). The publication date is the date of posting online at www.jneurosci.org.

http://www.jneurosci.org/cgi/content/full/5255 
liculus (IC) of the little brown bat that suggest that neural oscillation in combination with ordinary inhibition may be responsible for the creation of PLS, which has been shown previously to be important for temporal processing in these animals.

\section{MATERIALS AND METHODS}

Experimental subjects comprised six little brown bats, Myotis lucifugus. Details of experimental methods are given by Galazyuk et al. (2000). For surgery, the animal was anesthetized via halothane inhalation. After incision of the skin and clearing of the tissues above the skull, a small metal rod was glued to the skull. After the surgery, animals were allowed to recover for 2-4 $\mathrm{d}$ in individual holding cages.

Recordings were made from awake bats. During a recording session, the animal was placed inside a sound-attenuating chamber. The metal rod on the head of the bat was secured to a small holder to restrain the head of the animal atraumatically, leaving the ears unobstructed for free-field acoustic stimulation. A small hole $(\sim 50 \mu \mathrm{m})$ was then made in the skull overlying the IC, through which a recording electrode was inserted to reach the IC. Each recording session lasted 6-8 hr, and experiments proceeded every 2-3 d for a maximum of 4 weeks.

Extracellular single-unit recordings were made with glass micropipettes filled with $0.2 \mathrm{~m}$ potassium acetate. Recorded action potentials were amplified using standard audiovisual methods and processed offline. Our experimental protocols are in compliance with the Guide for the Care and Use of Laboratory Animals (publication number 86-23 of the National Institutes of Health) and with the Animal Welfare Act of 1966 and its amendments of 1970 and 1976. These protocols were reviewed and approved by the University of Illinois Laboratory Animal Use and Care Committee.

Acoustic stimuli, comprising tone pulses at the characteristic frequency $(\mathrm{CF})$ of the unit, were delivered to the bat via a free-field ultrasonic loudspeaker located $60 \mathrm{~cm}$ in front of the bat. Tone pulses had a constant rise-fall time of $0.5 \mathrm{msec}$ and a total duration of $2 \mathrm{msec}$. Sound pulses of a wide range of sound levels, from 10 to $90 \mathrm{~dB}$ sound pressure level (SPL), were presented at a rate of one per second in $4 \mathrm{~dB}$ (or $2 \mathrm{~dB}$ ) orderly increments. The range of sound level was extended to lower values if units responded to sound pulses at $10 \mathrm{~dB}$ SPL. The absolute sound pressure level was measured with a one-quarter-inch microphone situated near the concha of the ear opposite to the recording side. We normally presented 10-20 tone pulses at each sound level. Dot raster histograms were used to depict the temporal discharge pattern of a unit for each epoch at each level. Each dot in a raster histogram indicated a spike at the relative time instant with respect to the stimulus onset. Dot raster histograms from different sound levels were then combined to create a composite dot histogram of a unit so that how the response latency and the firing pattern changed globally with sound levels could be easily visualized. To quantify the response latency, we determined the average and the SD of the latency of the first spike of the responses of a unit to 10-20 epochs within the "response window" (defined as the window wherein the spike count and/or firing rate is $>25 \%$ above the background spontaneous firing). The spontaneous firing rate was the average response over a $50 \mathrm{msec}$ window before each stimulus epoch. The average latency at each sound level was used as a metric for assessing how the latency changed with sound amplitude.

For a number of IC neurons showing PLS, we applied a GABA receptor blocker, bicuculline $(10 \mathrm{~mm}, \mathrm{pH} 3.0)$, iontophoretically to evaluate the role of GABAergic inhibition in shaping the PLS and the temporal firing pattern of the unit. For this, a single-barrel recording pipette was attached to a five-barrel micropipette, with the tip of the single-barrel pipette protruding $\sim 20 \mu \mathrm{m}$ beyond the tip of the multibarrel pipette. When the drug was not being applied, a retention current of $-20 \mathrm{nA}$ was applied to the drug barrel to prevent drug leakage. To apply bicuculline, we used a depolarizing current of 5-20 nA. The response of a unit was evaluated before, during, and after drug application.

\section{RESULTS}

We studied the tone-pulse responses of 92 IC neurons having a wide range of CFs $(9-82 \mathrm{kHz})$ and thresholds at $\mathrm{CF}(5-84 \mathrm{~dB}$ SPL). In agreement with previous studies, practically all IC neurons showed level-dependent changes in their response latencies and/or spike count (Irvine, 1986; Heil and Irvine, 1997; Galazyuk et al., 2000; Klug et al., 2000). For 65 neurons (71\%), an increase
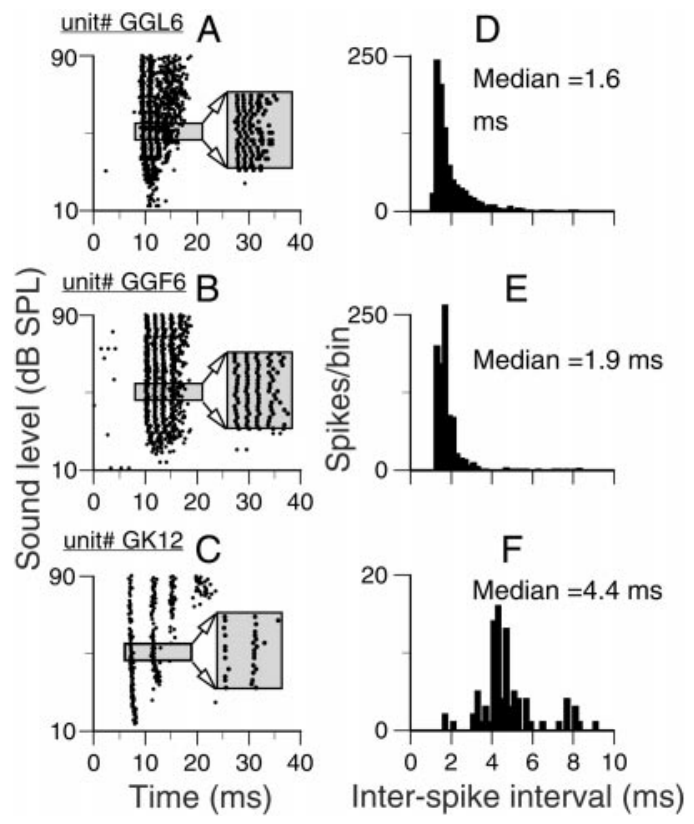

Figure 1. Composite dot raster histograms of three representative IC neurons in response to tone pulses at the CF of the unit (presented at a time of $0 \mathrm{msec}$ ) at sound levels from 10 to $90 \mathrm{~dB}$ SPL that display periodic firing patterns. Each dot represents a spike at a relative time instant (reference stimulus onset time). For each sound level, responses to 5 of the 10 trials are shown in the composite dot histograms. $A$, Unit GGL6 shows periodic discharges at 30-90 dB SPL with a dominant firing period of $1.6 \mathrm{msec}$; the firing periodicity of the unit can be observed by the composite interspike interval over the entire response range $(D)$. $B$, Same as $A$ but from unit GGF6 with a dominant firing period of $1.9 \mathrm{msec}$ (shown by the composite interspike interval in $E$ ). $C$, Same as $A$ but from unit GK12 with a dominant firing period of $4.4 \mathrm{msec}$ (shown by the composite interspike interval in $F$ ). Insets in $A-C$ show the expanded dot histograms at two sound levels, 48 and $52 \mathrm{~dB}$ SPL.

in sound amplitude elicited a systematic decrease in the latency of the first spike in the response of the unit (Fig. $1 A-C$ ). This shift was most pronounced at low sound levels, within $20 \mathrm{~dB}$ of the threshold of the unit at CF. The response latency did not change appreciably with an additional increase in sound level. The first spike latency was therefore a nonlinear function of SPL, as observed previously (Heil and Irvine, 1997). However, unlike the previous study, the first spike latency was also a nonlinear function of the maximum acceleration to peak pressure. The maximum overall shift in latency, between the highest and lowest sound amplitudes tested, ranged from 0.2 to $4.3 \mathrm{msec}$, with a mean of $1.35 \mathrm{msec}$; this was longer than $0.5 \mathrm{msec}$, the maximum shift in peak pressure resulting from amplitude change. For these neurons, an increase in sound amplitude concomitantly produced an increase in spike count.

For 17 of the 65 neurons, the increase in spike count occurred because of an increase in firing frequency and a marked increase in response duration (Fig. $1 A-C$ ). Interestingly, with one exception, an increase in sound level not only produced a longer response duration but the discharges also became periodic; the periodic firing patterns at high sound levels were readily observed in the composite dot histograms of the unit (Fig. $1 A-C$ ). The three units in Figure 1 fired one to two periodic cycles at 20-30 $\mathrm{dB}$ SPL, and the firing cycle grew to four to five at $\sim 50 \mathrm{~dB}$ SPL (Fig. 1, insets). For unit GGF6 in Figure $1 B$, its firing showed five periodic cycles at 76-90 dB SPL. Other IC neurons exhibited a maximum of two or three firing cycles. A noteworthy feature 
among these neurons was that the increase in firing duration at higher sound levels was attributed to the addition of later spikes. The firing duration could be as long as $15 \mathrm{msec}$, which was almost an order longer than the stimulus duration of $2 \mathrm{msec}$. The periodic discharge pattern of these neurons therefore appears to differ from that of chopper cells in the cochlear nucleus and other brainstem nuclei (Rhode and Greenberg, 1992), for which the chopping firing pattern is observed within the stimulation duration. However, studies of chopper cells have used much longer tone bursts at low sound levels, and thus it is unclear whether or not these cells would elicit sustained periodic firings in response to short tone pulses and at suprathreshold levels.

Another notable feature was that the firing periodicity was primarily level independent. This was evidenced by the following: (1) the stable interspike interval of the unit at each sound level between 30 and $90 \mathrm{~dB}$ SPL as shown by the vertical alignment of the first, second, and subsequent spikes for the three units in Figure 1; and (2) the tight cluster of the composite interspike interval histograms of the unit (Fig. $1 D-F$ ). In general, the later spikes showed greater jitters than earlier spikes (Fig. $1 A-C$, insets), and the jitter was more pronounced for units showing a longer firing period (Fig. 1C). In addition, within limits the firing periodicity was independent of stimulus frequency within $\pm 1 \mathrm{kHz}$ from CF (data not shown). The dominant firing periodicity for units GGL6, GGF6, and GK12 was 1.6, 1.9, and 4.4 msec, respectively. The firing periodicity was unit specific, ranging from 1.3 to $6.7 \mathrm{msec}$.

For the remaining 48 of the 65 neurons, an increase in sound level elicited an increase in spike count, but this was primarily attributable to an increase in firing rate without a notable increase in firing duration. These units typically fired a single short burst of action potentials over a wide range of sound amplitudes.

For 27 of 92 IC neurons (29\%), increasing the sound level elicited an increase in the latency of the first spike (i.e., these neurons exhibited PLS). In all but three PLS neurons, the latency shift occurred in quantum steps (Fig. 2). For 22 neurons, there was only a single discrete shift in the latency of the first spike over the range of 10-90 dB SPL (Fig. $2 A, C$ ). For example, unit GL26 showed a quantum shift in response latency from 14.4 to 19.7 msec with a change in sound level from $<46$ to $>54 \mathrm{~dB}$ SPL (Fig. $2 A, D)$. The latency shift for unit GB1 in Figure $2 C$ was smaller, from $16.2 \mathrm{msec}$ at $<46 \mathrm{~dB}$ SPL to $18.8 \mathrm{msec}$ at $>54 \mathrm{~dB}$ SPL (Fig. $2 F)$. The step size in latency shift was unit specific and ranged from 1.2 to $8.2 \mathrm{msec}$. We noted that the spike count was often sparse in the zone of latency transition, thereby giving rise to two or more discrete response ranges. Correspondingly, a change in sound level produced a large fluctuation in the spike count, with multiple peaks and valleys. Within each response range, the latency was relatively stable (Fig. $2 D-F$ ). Five units showed more than one step change in latency shift (Fig. $2 B$ ), and for them the multiple step sizes were essentially identical, as shown by the composite poststimulus (PST) histogram for unit GGJ5 (Fig. 2E).

For 14 of the 27 IC neurons showing PLS, we studied the responses of the unit to tone pulses before, during, and after iontophoretic application of bicuculline. Drug application increased the spike count of the unit as expected and abolished the PLS that was apparent during the predrug condition. Before drug application, unit GGK26 in Figure 3 responded to tone pulses falling within two intensity ranges with differential latencies (longer at the upper range); the latency of the first spike within each of the two ranges was relatively stable, centering at $\sim 14$ and $19 \mathrm{msec}$, respectively. With the administration of bicuculline,
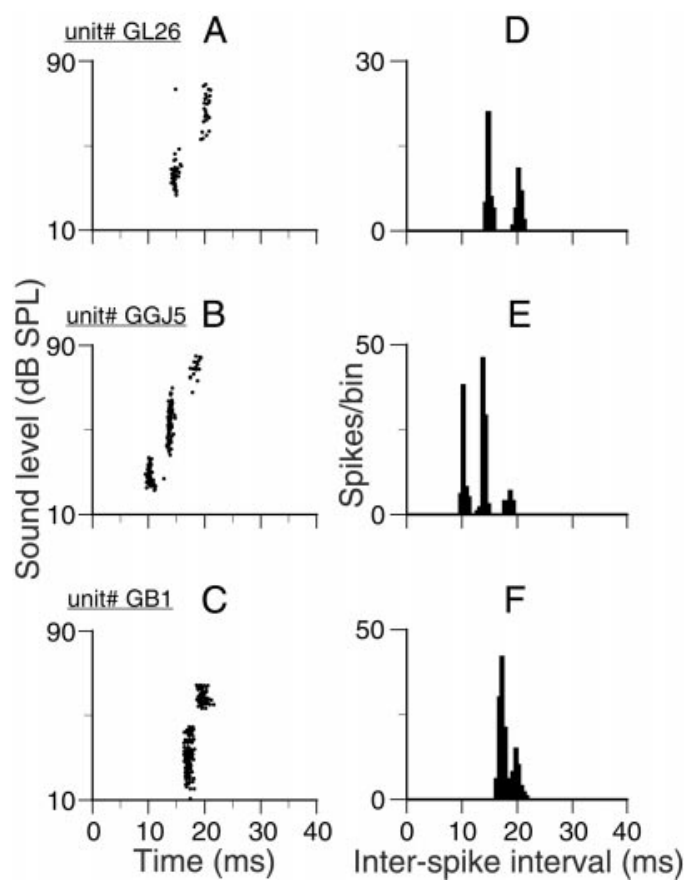

Figure 2. Composite dot raster histograms of three representative IC neurons in response to tone pulses at the CF of the unit at sound levels from 10 to $90 \mathrm{~dB}$ SPL that display PLSs. See the legend to Figure 1 for an explanation of a composite dot histogram. $A$, Unit GL26 shows a leveldependent shift in response latency of $5.3 \mathrm{msec}$; this quantum latency shift can be visualized from the composite PST histogram of the unit shown in $D$. The composite PST histogram was constructed by combining the responses of the unit across the entire range of sound levels with a bin width of 0.5 msec. $B$, Unit GGJ5 shows multiple shifts in response latency of $4.0 \mathrm{msec}$; its composite PST histogram is shown in $E$. $C$, Unit GB1 shows a level-dependent shift in response latency of $2.6 \mathrm{msec}$; its composite PST histogram is shown in $F$.

instead of responding to sound within two discrete intensity ranges with $\mathrm{PLS}$, the unit now responded over a broad range, from 18 to $90 \mathrm{~dB}$ SPL. At low sound levels (from 18 to $48 \mathrm{~dB}$ SPL), the latency of the first spike was only slightly advanced. Between 48 and $90 \mathrm{~dB}$ SPL, the firing of the unit was altered. An early response component $(\sim 14 \mathrm{msec})$ emerged before the response at $\sim 19 \mathrm{msec}$. Beyond $80 \mathrm{~dB}$ SPL, the firing pattern of the unit was once again modified with the addition of a later firing component $(\sim 23.5 \mathrm{msec})$. In other words, drug application transformed the temporal discharge pattern of the unit at high sound levels from phasic to periodic with multiple firing cycles. The average firing periodicity of $6 \mathrm{msec}$ closely approximated the level-dependent shift in response latency during the predrug period (Fig. 3B, composite PST histograms). At 5-10 min after bicuculline was withdrawn, the unit recovered from the drug effect and once again displayed PLS with a latency shift of $5 \mathrm{msec}$ (Fig. 3A,B, bottom panels). These results indicate that, in the intact condition, GABA acted to partially suppress, and therefore mask, the periodic discharges of these neurons.

The drug effects on level-dependent firing pattern and latency shift were similar in other IC neurons investigated. In 12 of 14 neurons, bicuculline abolished the PLS that was observed during the control period and produced periodic discharges at high sound levels. For each unit, the average firing periodicity observed during drug application approximated the average leveldependent shift in response latency during the predrug period (Fig. $3 C)$; these two values were tightly correlated $(r=0.89)$. 
Figure 3. Composite dot raster histograms $(A)$ and PST histograms $(B)$ of a representative IC neuron with PLS before (top row), during (middle row), and after (bottom row) iontophoretic application of bicuculline (20 $\mathrm{nA}$ ). See the legend to Figure 1 for other details. The composite PST histograms show the constancy of the firing periodicity of the unit across sound levels. Also, the PST histograms show that the level-dependent shifts in latency during the predrug and the recovery periods are matched and correlate closely with the firing periodicity of the unit during drug application. $C$, Summary data for 12 IC neurons showing that the average firing periodicity during drug application is correlated with the average level-dependent shift in response latency during the predrug period $(r=0.89)$.
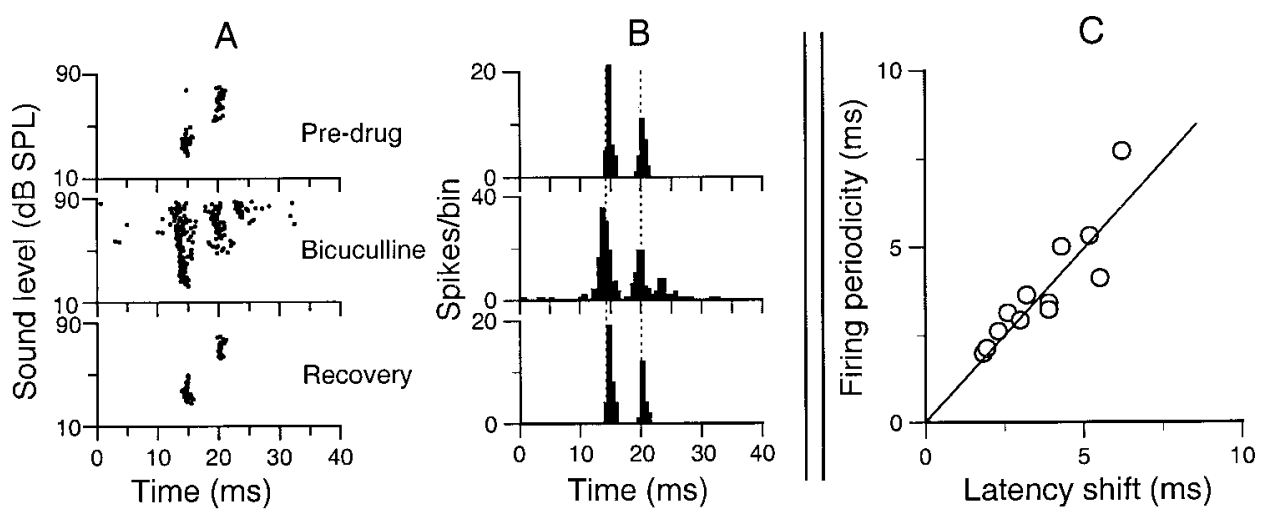

\section{DISCUSSION}

Results of our study reveal that 28 of 92 IC neurons investigated show evidence of prolonged oscillatory discharges at high sound levels. The first line of evidence is direct. Namely, 16 IC neurons exhibit periodic discharges in response to $2 \mathrm{msec}$ tone pulses at the CF of the unit with essentially constant firing periodicity. The periodicity induced by $2 \mathrm{msec}$ tone pulses is unit specific and relatively independent of stimulus frequency and intensity, suggesting that it may occur because of endogenous origins. Moreover, the duration of periodic firing is prolonged with an elevation of sound level, suggesting that the intrinsic oscillation is likely damped.

The second line of evidence from 12 IC neurons is indirect. For these neurons, the periodic discharge at high sound levels is evidenced only when GABAergic inhibition is suppressed (i.e., when bicuculline is administered). As before, the periodic discharge under bicuculline is unit specific and primarily independent of sound intensity, suggesting that its origin is likely endogenous. That the periodic discharge becomes progressively more robust at higher sound levels with more oscillatory cycles also suggests that the oscillation is damped.

Together, both sets of data are consistent in suggesting that these IC neurons have a tendency to discharge periodically because of intrinsic oscillatory mechanisms. Oscillation has been shown to occur widely in the nervous system (Steriade et al., 1990; Hutcheon and Yarom, 2000). In particular, studies in the visual system have shown that neurons at higher levels of the visual system often exhibit intrinsic oscillations and give rise to rhythmic discharges when optimally stimulated. In the auditory system, oscillatory potentials have also been observed in patch-clamp recordings from IC neurons of big brown bats (Covey et al., 1996); oscillatory potential can be observed in neurons having different CFs, across a wide range of stimulus amplitudes. Our observation is therefore consistent with the above study, as well as with results of extracellular studies in the IC (Grinnell, 1963; Suga, 1964). The extracellular studies indicate that IC neurons often show a periodic waxing and waning of responsiveness to the second of a pair of tone pulses separated by a variable interval.

Under intact conditions, 29\% of IC neurons exhibit PLS. This proportion is larger than the $12.8 \%$ reported for the IC of the Mexican free-tailed bat (Klug et al., 2000). This discrepancy may be attributed to a difference in methods. In the study by Klug et al. (2000), the dynamic range of acoustic stimulus was limited to $50 \mathrm{~dB}$; this is too narrow for observation of PLS occurring mostly at higher sound levels. The results of our pharmacological study in a subset of neurons exhibiting PLS are in agreement with those of Klug et al. (2000) showing that suppression of GABAergic inhibition abolishes PLS. Furthermore, our study reveals that PLS is attributed to partial inhibition of the oscillatory discharges of the unit. Our results therefore support the Timed Inhibitory model of Sullivan (1982a,b) but are at odds with his Multiple Delay Line model. However, the Timed Inhibitory model assumes that the inhibitory circuit produces a fixed delay that is determined by the duration of inhibition (Sullivan, 1982b, his Fig. 11). In contrast, our results show that the duration of inhibition is level dependent and not constant. At low sound levels, only the early spikes are inhibited; however, with increasing sound levels, additional later spikes may be suppressed. Level-dependent inhibition of the oscillatory discharges of the neuron conceivably contributes to multiple quantum latency shifts.

In light of our results, we advocate a radically different model for explaining PLS in IC neurons shown previously to be important for a delay-tuned response (Fig. 4). This model assumes that IC neurons (at least those showing PLS) have intrinsic resonances and that their membrane potentials show damped oscilla-
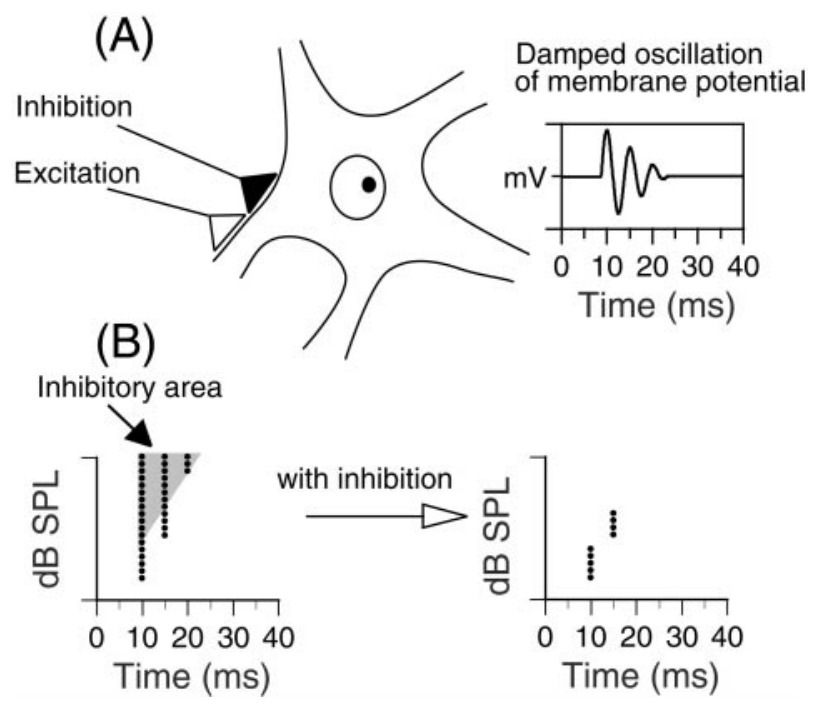

Figure 4. A working neural model for PLS. A, An IC neuron receiving both excitatory and inhibitory inputs. This cell shows endogenous damped oscillation of membrane potential when acoustically stimulated. $B$, Schematic dot raster histograms of the responses of the unit, at various sound levels without (left) and with (right) GABAergic inhibition; the inhibitory region is indicated by the shaded area. 
tions when cells are appropriately stimulated (Fig. 4A). This assumption is in line with results from our study and with a general view that all neurons have intrinsic resonances (Hutcheon and Yarom, 2000). This is because all cells have the following: (1) leaky resistance and capacitance that combine to serve as a low-pass filter, and (2) a potassium current that opposes lowfrequency fluctuations in membrane potential and therefore serves as a high-pass filter. Different phenotypes of ion channels can conceivably generate differential resonance frequencies, as shown for tonotopy in the auditory periphery. Another assumption is that these IC neurons receive both excitatory as well as inhibitory inputs that are level dependent. Because the oscillation in membrane potential is damped, low-level excitation would elicit only one (or two) spikes by the early components of the oscillating potential. The latency of this spike is not markedly affected by a small change in stimulus intensity. A large increase in intensity would elicit the firing of action potentials by the early as well as the later and damped components of oscillatory potential, thereby prolonging the oscillatory discharges but only when unmasked with bicuculline (Fig. $4 B$ ). When inhibition is present (e.g., in the intact case), the early spike is suppressed because of the transient inhibition, but the later spikes remain intact; therefore, the response latency is prolonged by a single oscillatory cycle (Fig. 4B). At still higher levels of stimulation, both the excitation and inhibition become stronger. The stronger excitation promotes firing of spikes by the later components of the oscillatory potential. At the same time, stronger inhibition (meaning longer-lasting inhibition) suppresses the second and/or the third spike in the train, prolonging the response latency even further (by another oscillatory cycle) or removing later spikes altogether. The relative strength of excitation and inhibition therefore determines whether the output neuron shows two or three dynamic response ranges with one or more quantum shifts in latency. In the extreme case, when inhibition is dominant and takes place even at low sound levels, the second and later spikes are suppressed altogether, leaving only the first spike that is observable over one narrow dynamic range. Strong inhibition therefore may create sharply tuned responses to sound amplitude.

The results of the physiological and pharmacological experiments reported herein are compatible with this model. However, at present there is no direct evidence for intrinsic resonances for neurons under study, and we cannot rule out the possibility that oscillation in IC neurons occurs because of oscillatory inputs from ascending afferents. Additional research is necessary to validate the model and elucidate the mechanisms underlying the delaytuned response.

\section{REFERENCES}

Covey E, Kauer JA, Casseday JH (1996) Whole-cell patch-clamp recording reveals subthreshold sound-evoked postsynaptic currents in the inferior colliculus of awake bats. J Neurosci 16:3009-3018.

Feng AS, Simmons JA, Kick SA (1978) Echo detection and targetranging neurons in the auditory system of the bat Eptesicus fuscus. Science 202:645-648.

Galazyuk AV, Llano D, Feng AS (2000) Temporal dynamics of acoustic stimuli enhance amplitude tuning of inferior collicular neurons. J Neurophysiol 83:128-138.

Grinnell AD (1963) The neurophysiology of audition in bats: intensity and frequency parameters. J Physiol (Lond) 167:38-66.

Heil P, Irvine DR (1997) First-spike timing of auditory-nerve fibers and comparison with auditory cortex. J Neurophysiol 78:2438-2454.

Hutcheon B, Yarom Y (2000) Resonance, oscillation, and the intrinsic frequency preferences of neurons. Trends Neurosci 23:216-222.

Irvine DRF (1986) The auditory brainstem. In: Progress in sensory physiology, Vol 7 (Autrum H, Ottoson D, Perl ER, Schmidt RF, Shimazu H, Willis WD, eds), pp 1-267. New York: Springer.

Klug A, Khan A, Burger RM, Bauer EE, Hurley LM, Yang L, Grothe B, Halvorsen MB, Park TJ (2000) Latency as a function of intensity in auditory neurons: influences of central processing. Hear Res 148:107-123.

Mittmann DH, Wenstrup JJ (1995) Combination-sensitive neurons in the inferior colliculus. Hear Res 90:185-191.

O'Neill WE (1995) The bat auditory cortex. In: Hearing by bats (Popper AN, Fay RR, eds), pp 416-498. New York: Springer.

O'Neill WE, Suga N (1979) Target range-sensitive neurons in the auditory cortex of the mustache bat. Science 203:69-73.

Rhode WS, Greenberg S (1992) Physiology of the cochlear nuclei. In: The mammalian auditory pathway: neurophysiology (Popper AN, Fay RR, eds), pp 94-152. New York: Springer.

Steriade M, Gloor P, Llinas RR, Lopes de Silva FH, Mesulam MM (1990) Basic mechanisms of cerebral rhythmic activities. Electroencephalogr Clin Neurophysiol 76:481-508.

Suga N (1964) Recovery cycles and response to frequency modulated tone pulses in auditory neurones in echolocating bats. J Physiol (Lond) 175:50-80.

Sullivan WE (1982a) Neural representation of target distance in auditory cortex of the echolocating bat Myotis lucifugus. J Neurophysiol 48:1011-1032.

Sullivan WE (1982b) Possible neural mechanisms of target distance coding in auditory system of the echolocating bat, Myotis lucifugus. J Neurophysiol 48:1033-1047. 